\title{
Image analysis techniques for the study of turbulent flows
}

\author{
Simone Ferrari ${ }^{1, *}$ \\ ${ }^{1}$ DICAAR - Dipartimento di Ingegneria Civile, Ambientale e Architettura, University of Cagliari, via Marengo 2, 09123 Cagliari, Italy
}

\begin{abstract}
In this paper, a brief review of Digital Image Analysis techniques employed in Fluid Mechanics for the study of turbulent flows is given. Particularly the focus is on the techniques developed by the research teams the Author worked in, that can be considered relatively "low cost" techniques. Digital Image Analysis techniques have the advantage, when compared to the traditional techniques employing physical point probes, to be non-intrusive and quasi-continuous in space, as every pixel on the camera sensor works as a single probe: consequently, they allow to obtain two-dimensional or three-dimensional fields of the measured quantity in less time. Traditionally, the disadvantages are related to the frequency of acquisition, but modern high-speed cameras are typically able to acquire at frequencies from the order of $1 \mathrm{KHz}$ to the order of $1 \mathrm{MHz}$. Digital Image Analysis techniques can be employed to measure concentration, temperature, position, displacement, velocity, acceleration and pressure fields with similar equipment and setups, and can be consequently considered as a flexible and powerful tool for measurements on turbulent flows.
\end{abstract}

\section{Introduction: Image Analysis}

Turbulence is present in almost every field of the human life and, consequently, it has many practical applications in Engineering, Medicine, Geophysics, etc. For instance, turbulent flows play a huge role in the motion of air around and behind cars, airplanes, boats, and other moving objects, because of drag and wake; in the reduction of the concentration of pollutants released in the atmosphere or in the sea (Ferrari and Querzoli, 2010 [1]) by human activities; in the weather forecasts, in the study of the motion of hurricanes, water and air masses in the ocean and in the atmosphere; in the optimization of mixing and combustion and in flow control (Rossi et al 2009 [2]) in engines and chemical reactors; in biological flows such as the air flowing through the lungs (Ramuzat and Riethmuller, 2001 [3]) or the blood motion in the aorta (Elkins et al., 2005 [4]) or downstream a stenosis (Zhu et al., 2011 [5]). The scalar quantity transport by turbulent flows plays a relevant role in many environmental phenomena, such as particle transport in geophysical flows (Boffetta et al., 2000 [6]). Following Meng et al (2016 [7]), turbulence is also one of the key phenomena in the study of the process of flotation for the separation of minerals. Moreover, the turbulent flow conditions caused by the motion of a swimmer in water are studied to predict the hydrodynamic forces and the swimmer's potential speeds (Takagi et al 2016 [8]). Turbulence plays also a fundamental role in the air quality and human comfort in built environment (Blocken et al, 2016 [9], Fu et al 2015 [10]).

Even if the computational capabilities of modern computers are always improving, the direct numerical simulation of high Reynolds number turbulent flows is still very difficult. Consequently, the role of experimental measures in the laboratory are at this stage still essential.

Traditional systems to perform measures in the laboratory are the single point probes, such as resistive probes to measure concentrations or Laser-Doppler Velocimetry (LDV, also known as Laser Doppler Anemometry LDA), Pitot tubes, Hot Wire Anemometer (HWA), Hot-film Anemometer (HFA) and Pulsed Wire Anemometer (PWA) to get the time history of velocity in a single point of space. They have the advantage of a high acquisition frequency (order of $10^{5} \mathrm{~Hz}$ ) but in order to obtain a field of the measured quantity, a number of probes high enough must be used or, when the experiment is repeatable, at the end of each run they can be moved to another position and the experiment repeated many times. On one hand, as the single point probes are intrusive (i.e. they alter the flow they should measure), it is nearly impossible to sample simultaneously many points in the flow, particularly if they are close to each other. On the other hand, the repetition of an experiment for as many times as the number of points needed to well define in space the investigation area can be very time-consuming. Moreover, a hot probe induces thermal convection on the flow, causing another local perturbation. A non-intrusive technique is the Laser Doppler Anemometry (LDA) that is still a point-wise technology. All these techniques perform measures in a fixed point in space, so they allow to obtain only Eulerian information. As stated by Cao et al (2014 [11]), to obtain accurate and detailed measures of the global turbulent airflow field by means of pointwise velocimetry is still excessively difficult and timeconsuming.

*Corresponding author: ferraris@unica.it 
For this reason, optical techniques, such as Digital Image Analysis (DIA) one, have gradually become alternative tools for measuring turbulent flow quantities. In this techniques, a camera with one or more sensors (CCD - Charge-Coupled Device or CMOS (Complementary Metal-Oxide-Semiconductor) shoots images of the flow, previously seeded with non-buoyant particles or dyes and lighted by a light source, at a given frequency. The first advantages of DIA techniques are that they are non-intrusive, quasi-continuous in space (as every pixel on the camera sensor works as a single probe): consequently, they allow to obtain twodimensional or three-dimensional fields of the measured quantity in less time, if compared with single point probes. Moreover, even if the directly measured quantity is the light intensity, many quantities can be measured indirectly, such as the position or the size of objects in the investigation area, concentration of substances, temperature, or, when comparing two consecutive images, the displacement, velocity and acceleration of moving objects. Moreover, the pressure on solid interface or in the flow filed can be measured as well.

Another advantage of DIA techniques is that some of them give access to Lagrangian information. Moreover, they can be performed employing relatively low cost equipment. Traditionally, the disadvantages of DIA techniques are related to the frequency of acquisition, but modern high-speed cameras are typically able to acquire at frequencies from the order of $1 \mathrm{KHz}$ to the order of $1 \mathrm{MHz}$. A disadvantage that, on the contrary, cannot be overtaken is that an optical access to the region of interest is needed, so transparent materials must be employed. Moreover, a certain care must be given to the index of refraction, in particular with curve surfaces.

In the following chapters, a brief review of Digital Image Analysis techniques employed to measure various quantities relevant for the study of turbulent flows is given, with a particular focus on the techniques developed by the research teams the Author worked in, that can be considered relatively "low cost" techniques.

\section{Concentration}

DIA techniques to measure the concentration of a certain substance in a flow is based on the characteristic of some dyes, in given quantities, to absorb or radiate light. In the first case, we have Light attenuation (LA), in the second case Light/Laser Induced Fluorescence (LIF).

\subsubsection{Light Attenuation}

The Light Attenuation (LA) technique has been used since the 1990s and it is based on the fact that the light from the back-lighting is attenuated (absorbed) by the dye as it passes through the flow, so the light source is typically opposite to the camera. Allgayer and Hunt (2012 [12]) has described the application of LA, focusing on the selection of chemical dye, dye concentration, illumination and optics. A typical dye for LA is the methylene blue. Anyway, a suitable dyeing agent for LA has to be a strongly light-absorbing substance (even at low concentrations) which does not react with the fluid in which it is dissolved. As the light is passing throw the flow, the measured concentration is usually the integral of the concentration in the direction of the light.

Hacker et al (1996 [13]) employed LA to determine the mixing of fluid properties transported by gravity currents.

Choi et a. (2016 [14]) has studied with this technique the layer-averaged concentration fields in of an inclined buoyant jet discharged into a co-flowing current

\subsubsection{Laser Induced Fluorescence}

The Light/Laser Induced Fluorescence (LIF or PLIF, i.e. Planar LIF) has been employed since the 1970s to measure concentration of certain substances in fluid flows (e.g., Stewart and Judeikis, 1974 [15]). The principle is the flow is seeded with a dye (such as Fluorescein or Rhodamine, see Melton and Lipp -2003 [16]- for a review of the techniques, dyes and criteria for quantitative PLIF measures) that, when meet an incident light at a certain frequency, radiates light at a different frequency. Provided the density of the substance is in the correct range, a linear relation between the intensity of the emitted light and the concentration of the dye holds. The dye concentration has usually to be very low, in order to avoid nonlinearity between light intensity and dye concentration and to minimize the light attenuation (Sutton et al. 2008 [17]). So in LIF a light/laser sheet illuminates the region of interest (typically, a plane section of the flow) perpendicularly to the camera, in order that the flow itself results on the image as a light flow on a dark background. When a laser light is not available, the light sheet can be generated employing one or more slide projectors, each one equipped with a black slide with narrow, vertical slit at its centre and focused in the observational area (see Ferrari and Querzoli -2010 [1]- for more details): in this way, a very inexpensive set-up to perform quantitative measurements of flow density can be obtained. A reference image of the lighted background has to be acquired to measure the distribution of the light intensity in the observational area: in this way, the intensity of the fluorescent light varies only because of the incident light. Filters on the camera lens can be employed to filter out all the frequencies but the one coming from the dye, even if this reduces the amount of light recorded by the camera sensors.

Ferrari and Querzoli (2010 [1]) employed this set-up to measure the dilution of the salt (directly proportional to the fractional volume of the fluorescein) in a negatively buoyant jet released in a stagnant environment.

A similar set-up was then employed to measure the same phenomenon released in a wavy environment (Ferrari and Querzoli 2015 [18]).

Bouche et al (2012 [19]) studied the mixing in a swarm of bubbles rising in a confined cell using a PLIF employing two different dyes. 
Techniques that couple LIF and Particle Image Velocimetry (PIV, see hereafter), allowing to obtain simultaneous measures of concentration and velocity, have been developed (e.g., Charogiannis et al 2015 [20]) using a two-cameras set-up.

\section{Temperature}

A typical DIA technique to measure the temperature in a flow is the use of temperature sensitive particles (TSPs), able to change colour with temperature, to put them into the flow and to record the flow with a camera. This allows the simultaneous and non-intrusive measurement of temperature and velocity (e.g. with Particle image velocimetry PIV or Particle Tracking velocimetry, see beyond) fields. For instance, Massing et al., 2016 [21], obtained the temperature fields from the intensity (thermographic phosphors) or frequency of the light emitted or reflected by the tracer particles (polymer particles doped with the temperature sensitive dye europium III thenoyltrifluoroacetonate-EuTTA- and the nearly temperature insensitive reference dye perylene).

Someya et al., 2010 [22], employed phosphor thermometry to visualize temperature fields (luminophors, including phosphors, are typically rare earth-doped ceramics that emit light when suitably excited).

Also Temperature Sensitive Paint (TSP) techniques, providing spatial temperature distributions on surfaces, can be employed. For instance, Ozawa (2016 [23]), employed TSP to experimentally study the unsteady aerothermodynamic phenomena occurring on a shocktube wall.

Moreover, thermal camera con be used, as done by Sagaidachnyi et al (2016 [24]), who employed cooled thermal imaging cameras to estimate the blood flow oscillations at the surface of living body

\section{Position}

If the flow is seeded with an emitting light dye, in addition to concentration, the position of a lighted fluid or particle on a dark flow or background can be detected with a DIA technique. For instance, Ferrari et al (2016 [25]), evaluated the wave motion transformations above a breakwater, both submerged and emerged, with a DIA technique. The fluid was seeded with a fluorescent dye, and the free surface was identified with a robust algorithm, that showed to properly work also in prohibitive situations for traditional resistive probes (e.g., very shallow waters and/or breaking waves) and to be able to measure the free surface all over the investigation field in a non-intrusive way.

Mendez et al (2016 [26]) measured in a similar way the thickness of a vertical falling liquid film, as well as the wave celerity and the wave profile.

Brady et al (2009 [27]) gave a review of different methods for particle image sizing, with a particular regard to the improvement of accuracy and robustness. This measure is quite relevant in multi-phase flows, that can be encountered in various industrial applications (e.g., the atomization of droplets in sprays, particulate flows, powders, fluidization, bubbly flows, flotation, etc.), where the features of the flow can be determined by the particle distribution and by the interaction of them with the fluid.

\section{Displacement}

If the position of an object, such a particle, can be spotted in an image, its displacement between two images taken at different times can be measured and, consequently, the trajectory of the object in time can be reconstructed. Moreover, dividing the displacement by the interval time, an indirect measure of the velocity can be attained. If the measure is good enough, the object acceleration can be measured as well.

\subsection{Velocity}

DIA techniques suitable for velocity measurement can be basically classified as Particle Streak Velocimetry (PSV), Particle Tracking velocimetry (PTV), Particle Image Velocimetry (PIV) and Feature Tracking (FT). All these techniques evaluate the velocity measuring the distance covered by a feature (a particle or a marker for PSV, PIV and PTV, a point of high gradient of the intensity for FT) on the images in a given time.

These DIA techniques for velocimetry give measures all over the investigation field (or at least where particles/features are found), do not need a probe (so they are essentially not invasive, at least if the particles are non-buoyant) and do not need any particular calibration (as the relation between the measured quantity, a displacement, and the required one, a velocity, is linear).

In all these techniques, the set-up consists of a fluid seeded with neutrally buoyant particles, illuminated with a light source and of one or more cameras shooting pictures at known temporal frequency.

The relevant scientific and technical progress attained in the last years in lasers, cameras, postprocessing, velocimetry codes and computer capabilities has led to the widespread diffusion of DIA velocimetry techniques.

\subsubsection{Particle Streaks Velocimetry}

In Particle Streak Velocimetry (PSV) the velocity is measure along the streaks left by moving particles on a long exposure image (e.g., Bergthorson and Dimotakis, 2006 [28]).

In this technique, the ambiguity between particles (typical of PIV or PTV) can be reduced, but strong issues can arise when particles enter or exit the investigation area or when particle trajectories have a strong curvature.

Moreover, ambiguity on the particle direction can arise. 


\subsubsection{Particle Tracking Velocimetry}

In Particle Tracking Velocimetry (PTV), the particle centroid is identified and tracked from one image to the following one (e.g. Dalziel, 1992 [29], Querzoli, 1996 [30], Virant and Dracos, 1997 [31]). In this way, PTV allows to reconstruct trajectories and to attain a Lagrangian measure and it differs from Particle Image Velocimetry (PIV) because this last relies on pattern matching in an essentially Eulerian way.

PTV information achieve a high local accuracy in every seeded point of the flow, with the possibility of solving small velocity variations and of obtaining a higher spatial resolution compared to PIV; on the other side, PTV is much more sensitive to noise. Moreover, a Eulerian description can be extracted from the Lagrangian information if there are enough samples to cover the investigation field. On the opposite, it is not possible to get reliable Lagrangian information starting from PIV data, because the transformation from the Eulerian description to the Lagrangian one implies an integration in time of the velocity field, not very reliable especially when considering turbulent flows with high sensitivity to initial conditions.

In PTV, the density of particles has to be low enough to allow the recognition of the single particles.

The main drawbacks of PTV are linked to the particle appearing/disappearing, to the difficulties in distinguishing overlapping particles and o the amount of data needed to cover the whole investigation field. So, PTV is complementary to PIV, with its ability to cope with low and medium particle concentration, to reach a higher spatial resolution and to cope with specific cases like regions with strong velocity gradients.

PTV shows clear advantages to PIV in near-wall regions with high velocity gradients as they can provide measurements nearest to the wall (Stanislas et al., 2005 [32]).

After a decade where PIV was often preferred, as it was easier to obtain results than with PTV, this last is becoming again popular, in particular with the development of three-dimensional measurements.

As stated by Fu et al (2015 [10]) in their review of PTV and PSV techniques for indoor airflow field investigation, the PTV technique has gradually become again a powerful tool, as the quantitative and detailed turbulent flow information obtained by PTV measurement is critical for analysing turbulent properties and developing numerical simulations.

As a matter of fact, one of the conclusions of the last PIV Challenge has been that sophisticated PTV algorithms can outperform state-of-the-art PIV algorithms (Kahler et al 2016 [33]).

\subsubsection{Particle Image Velocimetry}

The term Particle Image Velocimetry (PIV) first appeared in the literature in 1980s: reviews can be found by Adrian, 1991 [34], Buchave, 1992 [35], Adrian, 2005 [36], Adrian and Westerweel, 2011 [37]. An extensive review of the PIV techniques employed for the experimental wind comfort assessment at pedestrian level has been done in Blocken et al (2016 [9]).

In PIV, a spectral analysis or an auto/cross correlation is performed on a small interrogation window on two consecutive images in order to get a single velocity for each interrogation window. PIV algorithms obtain velocity fields comparing windows of successive frame on a regular grid and maximizing the correlation function to obtain their displacement. PIV is consequently fairly robust to noise and has a spatial resolution that is inversely proportional to the interrogation window size.

PIV allows higher seeding levels when compared with PTV.

As stated by Kahler et al. (2016 [33]), in the last decade, worldwide PIV development efforts have resulted in significant improvements in terms of accuracy, resolution, dynamic range and extension to higher dimensions.

As stated by Cao et al. (2014 [11]), PIV has gradually become the most popular and promising technique for airflow field measurement in indoor environment during the last decade. The main reasons for this are the well-developed technologies, a lot of experimental experiences and a large availability of commercially codes and systems.

For resolving the directional ambiguity, Stucky et al (1994 [38]) employed a two-colour PIV system: two differently coloured sheets of light illuminate the flow field at a different time, in order to distinguish the first image from the second image. Filters are employed to separate the two colours during interrogation, thus creating two images that are then cross-correlated with each other.

Falchi et al. (2006 [39]) has proposed a Robust Image Velocimetry (RIV), where the measure of the dissimilarity between interrogation windows is employed (instead of the auto/cross correlation typical of traditional PIV) which is based on a more robust estimator than cross-correlation.

\subsubsection{Feature Tracking}

In Feature Tracking (Lucas and Kanade, 1981 [40], Shi and Tomasi, 1994 [41], Miozzi et al, 2008 [42]) the object or region to be tracked is not chosen a priori, like in PTV and PIV, but by the method itself, as a feature that can be well tracked: a certain number of points in the picture with a high spatial gradients of light intensity are usually selected.

As stated by Miozzi et al. (2008 [42]), the displacements are obtained by searching the features between the first and second image, in a way which minimize a correlation distance between them (the minimum of the sum of squared differences of interrogation windows intensities). The linearized equation governing the minimization problem is solved in an iterative way only where the solution is guaranteed to exist. FTV provides the velocity in a Lagrangian framework. 


\subsubsection{Hybrid methods}

Some hybrid schemes (e.g. the one by Cowen et Monismith, 1997 [43]) combining PIV and PTV have been proposed.

Besalduch et al. (2013 [44] and 2014 [45]) has developed and tested a Feature Tracking Velocimetry (FTV), attempting to combine in a single scheme the advantages of the previous presented techniques. FTV compares windows only where the motion detection may be successful (as in FT, where there are high luminosity gradients in two directions, i.e. corners) FTV procedure consist of:

- identification of the features using the Harris corner detection (Harris \& Stephens, 1988 [46]);

- ordering of the features according to their cornerness;

- selection of the first $\mathrm{N}$ features and computation of the velocity by the comparison a window centered with the $i^{\text {th }}$ feature $\left(\mathrm{W}_{\mathrm{t}}\right)$ with windows $\left(\mathrm{W}_{\mathrm{t}+1}\right)$ within a range of displacements, $\left(\mathrm{d}_{\mathrm{i}}, \mathrm{d}_{\mathrm{j}}\right)$ in the next frame;

- for each displacement, a measure of the dissimilarity, $\mathrm{D}\left(\mathrm{d}_{\mathrm{i}}, \mathrm{d}_{\mathrm{j}}\right)$, between $\mathrm{W}_{\mathrm{t}}$ and $\mathrm{W}_{\mathrm{t}+1}\left(\mathrm{~d}_{\mathrm{i}}, \mathrm{d}_{\mathrm{j}}\right)$ is computed using the Lorentzian estimator and the displacement is consequently minimizing the dissimilarity;

- displacements are validated by means of algorithm based on Gaussian filtering of first neighbors (defined by the Delaunay triangulations), and simulated annealing.

FTV has shown to be suitable for measurements on a wide range of seeding density (from the high levels of PIV to the low ones of PTV), for the analysis of images with intermediate seeding density or non-homogeneous seeding and in presence of different seeding density.

\subsection{Acceleration}

As the material acceleration in a fluid flow is an intrinsically Lagrangian quantity, being the sum of a local (Eulerian) acceleration and of a convective acceleration, it cannot be reliably derived from traditional PIV but has to be derived from PTV Lagrangian measurements. Unfortunately, as before stated, PTV is very sensitive to measurement noise, which tends to grow at every derivation in time (from displacement to velocity to acceleration).

To reduce this problem, low-pass filter on the tracked particle positions have been proposed (e.g., La Porta et al, 2001 [47], Voth et al., 2002 [48], Mordant et al., 2004 [49]). The main parameter is the temporal window size of the filter: on one side, when it is too large, small fluctuations of the measured values can be deleted; on the other, when it is too small, the noise is not removed.

Ferrari and Rossi (2008 [50]) has developed a selfadaptive iterative method (Particle Tracking Velocimetry and Accelerometry, PTVA) that relies on an adaptive number of tracked positions, for the trajectories approximation, depending only on the local flow properties. The criterion proposed holds the potential to withdraw some convection/sweeping information and thus to extract more accurately the acceleration.

In a first step, the position extracted by PTV are approximated into the trajectory components via a least square approximation, making use of a fourth order moving polynomial, with a variable number of positions depending only on a minimum and maximum: from the temporal derivatives of this approximated trajectory components, a first guess velocity components are computed via a temporal derivative.

In the second step, the same approximation is performed starting again from the positions extracted by PTV, again using the least square approximation of a fourth order moving polynomial, but with a criterion that adapts the number of positions for the approximation in order to have a parameter (called the convected displacement), able to take into account the local flow properties, as close as possible to a target value, equal to the perimeter of the smallest eddy to be measured. The components of the velocity and acceleration are then extracted via temporal derivatives.

From the comparison with methods using a constant number of positions, PTVA errors are about six times smaller for velocity and about four times smaller for acceleration. The high quality of this acceleration measurement allowed to even measure the divergence of the acceleration, strictly related to the stirring in the flow.

PTVA has been applied to extract the single terms of the Navier-Stokes equations (Lardeau et al., 2009 [51]), for flow control purposes (Rossi et al. 2010 [2]) and to investigate time dependent quasi-two-dimensional flows (Ferrari et al., 2007 [52] and 2008 [53]).

Ultrasonic techniques employing fast silicon strip detectors have been used to optically image short particle (tiny transparent beads) tracks and measure particle acceleration (La Porta et al., 2001 [47], Voth et al, 2002 [48]), reaching a very high time resolution (up to 70,000 frames per second) but in a small measurement volume (particles can be followed for only few Kolmogorov time scales). The technique is based on the capability to timeresolve the Doppler shift of the sound scattered by continuously insonified particles.

\section{Pressure}

Traditional techniques for pressure measurement away from solid boundaries are usually based on Pitot-tube type of probes (as previously stated, intrusive and pointwise).

Another technique used in the past to measure pressure away from the boundaries was based on microscopic bubbles as pressure sensors (Ran and Katz, 1994 [54]), but they provided only a very limited number of data points for each run of measure.

DIA techniques have reached a level of development that allows to even measure the pressure fields in a flow.

For instance, the PTVA technique (Ferrari and Rossi 2008 [50]), allowing to measure all the terms in the Navier-Stokes equation, gives also access to the pressure gradient term all over the investigation field. 
Another non-intrusive technique for measuring the instantaneous spatial pressure distribution in a flow field is the four-exposure PIV system developed by Liu and Katz (2006 [55]): they measure the distribution of the material acceleration by comparing the velocity of the same group of particles at different times and then integrating it to obtain the pressure distribution. Exposing both cameras to the same particle field at the same time and cross-correlating the images enables precision matching of the two fields of view.

A last technique is the Pressure Sensitive Paint (PSP), a non-intrusive optical pressure measurement technique that provides spatial pressure distributions but only on surfaces of solid objects. Gregory et al. (2014 [56] have reviewed fast-responding PSP techniques for applications to aerodynamic and acoustic investigations. PSP determines surface pressure distributions by measuring changes in the intensity of emitted light, while fast-responding PSP is an extension applicable to unsteady flows and acoustics. Most fast-responding PSP paints are based on the development of porous binders allowing a fast oxygen diffusion and interaction with the chemical sensor.

\section{Conclusions}

Digital Image Analysis techniques for the measure of different quantities in turbulent flows have been briefly reviewed. The relevant scientific and technical progress achieved in recent years in lasers, cameras, postprocessing, velocimetry codes and computer capabilities has taken to the widespread diffusion of DIA techniques. They have the advantage, when compared to the traditional techniques employing physical point probes, to be non-intrusive and quasi-continuous in space. DIA techniques can be employed to measure concentration, temperature, position, displacement, velocity, acceleration and pressure fields with similar equipment and setups, and can be consequently considered as a flexible and powerful tool for measurements on turbulent flows.

The Author would like to acknowledge Prof. Maria Grazia Badas, Dr. Luigi Antonio Besalduch, Mr. Antonio Mascia, Prof. Giorgio Querzoli, Dr. Lionel Rossi and Prof. John Christos Vassilicos.

\section{References}

1. Ferrari, S., Querzoli, G., Mixing and re-entrainment in a negatively buoyant jet, Journal of Hydraulic Research, 48 (5), pp. 632-640. (2010)

2. Rossi, L., Bocquet, S., Ferrari, S., Garcia de la Cruz, J.M., Lardeau, S., Control of flow geometry using electromagnetic body forcing, International Journal of Heat and Fluid Flow, 30 (3), pp. 505-513. (2009)

3. Ramuzat, A., Riethmuller, M.L. Steady and unsteady PIV investigations of flows within a 3D lung bifurcation model, DLR-Mitteilung, (3), pp. 109-119 (2001)
4. Elkins, C.J., Iyengar, A.S., Draney, M.T., Dake, M.D., Medina, F., Wicker, R.B. Rapid in-vitro MRV and PIV measurements in anatomically accurate human thoracic aorta phantoms, Proceedings of the 2005 Summer Bioengineering Conference, 2005, pp. 235-236 (2005)

5. Zhu, H., Qian, M., Zou, Y., Song, R., Niu, L., Jiang, B., Guang, Q., Zheng, H. The validation of EchoPIV technique used in a stenosis model, 5th International Conference on Bioinformatics and Biomedical Engineering, iCBBE 2011, art. no. 5780292 (2011).

6. Boffetta G., Celani A. and Vergassola M., Inverse energy cascade in two-dimensional turbulence: deviation from Gaussian behaviour, Physical Review E, 61 (1), R29-R32, (2000).

7. Meng, J., Tabosa, E., Xie, W., Runge, K., Bradshaw, D., Manlapig, E. A review of turbulence measurement techniques for flotation, Minerals Engineering, 95, pp. 79-95 (2016)

8. Takagi, H., Nakashima, M., Sato, Y., Matsuuchi, K., Sanders, R.H. Numerical and experimental investigations of human swimming motions, Journal of Sports Sciences, 34 (16), pp. 1564-1580, (2016).

Blocken, B., Stathopoulos, T., van Beeck, J.P.A.J. Pedestrian-level wind conditions around buildings: Review of wind-tunnel and CFD techniques and their accuracy for wind comfort assessment, Building and Environment, 100, pp. 50-81 (2016).

9. Fu, S., Biwole, P.H., Mathis, C. Particle tracking velocimetry for indoor airflow field: A review, Building and Environment, 87, pp. 34-44 (2015).

10. Cao, X., Liu, J., Jiang, N., Chen, Q. Particle image velocimetry measurement of indoor airflow field: A review of the technologies and applications, Energy and Buildings, 69, pp. 367-380 (2014).

11. Allgayer D, Hunt GR. - On the application of the light attenuation technique as a tool for non intrusive buoyancy measurements, Exp. Thermal Fluid Science 38, 257-261 (2012).

12. Hacker J, Linden PF, Dalziel SB. Mixing in lockrelease gravity currents, Dynamics of Atmospheres and Oceans 24, 183-195 (1996).

13. Choi, K.W., Lai, C.C.K., Lee, J.H.W. Mixing in the intermediate field of dense jets in cross currents, Journal of Hydraulic Engineering, 142 (1), art. no. 04015041 (2016).

14. Stewart, T.B., Judeikis, H.S. Measurements of spatial reactant and product concentrations in a flow reactor using laser induced fluorescence, Review of Scientific Instruments, 45 (12), pp. 1542-1545 (1974)

15. Melton LA, Lipp C.W., Criteria for quantitative PLIF experiments using high-power lasers, Experiments in Fluids 35, 310-316 (2003)

16. Sutton, J.A., Fisher, B.T., Fleming, J.W. A laser induced fluorescence measurement for aqueous fluid flows with improved temperature sensitivity. Exp. Fluids 45(5), 869-881 (2008).

17. Ferrari, S., Querzoli, G., Laboratory experiments on the interaction between inclined negatively buoyant 
jets and regular waves, EPJ Web of Conferences, 92, art. no. 02018 (2015)

18. Bouche E., Cazin S., Roig V., Risso F and Billet A.M., Mixing in a swarm of bubbles rising in a confined cell measured by mean of PLIF with two different dyes, 16th Int Symp on Applications of Laser Techniques to Fluid Mechanics Lisbon, Portugal, 09-12 July, 2012

19. Charogiannis, A., An, J.S., Markides, C.N. A simultaneous planar laser-induced fluorescence, particle image velocimetry and particle tracking velocimetry technique for the investigation of thin liquid-film flows. Experimental Thermal and Fluid Science, 68, pp. 516-536 (2015).

20. Massing, J., Kaden, D., Kahler, C.J., Cierpka, C. Luminescent two-color tracer particles for simultaneous velocity and temperature measurements in microfluidics, Measurement Science and Technology, 27 (11) (2016).

21. Someya S., Tominaga K, Li Y, Okamoto K, Combined velocity and temperature measurements of natural convection using temperature sensitive particles, 15th Int Symp on Applications of Laser Techniques to Fluid Mechanics Lisbon, Portugal, 05-08 July, 2010

22. Ozawa, H. Experimental study of unsteady aerothermodynamic phenomena on shock-tube wall using fast-response temperature-sensitive paints, Physics of Fluids, 28 (4) (2016)

23. Sagaidachnyi, A.A., Volkov, I.U., Fomin, A.V. Influence of temporal noise on the skin blood flow measurements performed by cooled thermal imaging camera: Limit possibilities within each physiological frequency range, Progress in Biomedical Optics and Imaging - Proceedings of SPIE, 9917, art. no. 99170N (2016).

24. Ferrari, S., Badas, M.G., Querzoli, G., A nonintrusive and continuous-in-space technique to investigate the wave transformation and breaking over a breakwater, EPJ Web of Conferences, 114, art. no. 02022 (2016).

25. Mendez, M.A., Németh, L., Buchlin, J.-M. Measurement of Liquid Film Thickness via Light Absorption and Laser Tomography, EPJ Web of Conferences, 114, art. no. 02072 (2016)

26. Brady, M.R., Raben, S.G., Vlachos, P.P. Methods for Digital Particle Image Sizing (DPIS): Comparisons and improvements, Flow Measurement and Instrumentation, 20 (6), pp. $207-$ 219 (2009).

27. Bergthorson J. M. and Dimotakis P. E., Particle velocimetry in high-gradient/high-curvature flows, Experiments in Fluids, 41 (2), 255-263, (2006).

28. Dalziel S.B., Decay of rotating turbulence: some particle tracking experiments, Applied Scientific Research, 49, 217-244, (1992).

29. Querzoli G, A Lagrangian study of particle dispersion in the unstable boundary layer. Atmos Environ 30(16):2821-2829 (1996)

30. Virant M. and Dracos T., 3D PTV and its application on Lagrangian motion, Measurement Science and Technology, 8, 1539-1552, (1997).
31. Stanislas M., Okamoto K., Kähler C.J. and Westerweel J., Main results of the Second International PIV Challenge, Experiments in Fluids, 39, 170-191, 2005.

32. Kähler, C.J., Astarita, T., Vlachos, P.P., Sakakibara, J., Hain, R., Discetti, S., La Foy, R., Cierpka, C. Main results of the 4th International PIV Challenge, Experiments in Fluids, 57 (6) (2016)

33. Adrian R.J., Particle-imaging techniques for experimental fluid mechanics, Annual Review of Fluid Mechanics, 261-303, (1991).

34. Buchhave, P. Particle image velocimetry-status and trends, Experimental Thermal and Fluid Science, 5 (5), pp. 586-604 (1992).

35. Adrian R.J., Twenty years of particle image velocimetry, Experiments in Fluids, 39 (2) 159-169 (2005)

36. Adrian R.J., Westerweel J., Particle image velocimetry. Cambridge (2011)

37. Stucky, M.J., Nino, E., Gajdeczko, B., Felton, P.G. Two-color particle image velocimetry technique for an internal combustion engine, Experimental Thermal and Fluid Science, 8 (4), pp. 305-314 (1994)

38. Falchi, M., Querzoli, G., Romano, G.P. Robust evaluation of the dissimilarity between interrogation windows in image velocimetry, Experiments in Fluids, 41 (2), pp. 279-293 (2006)

39. Lucas B.D. and Kanade T., An Iterative Image Registration Technique with an Application to Stereo Vision, Proceedings of Imaging Understanding Workshop, Washington, D.C. (USA), 121-130, 1981.

40. Shi J. and Tomasi C., Good features to track, Proceedings of the IEEE Conference on Computer Vision and Pattern Recognition (CVPR94), Seattle, USA, 1994.

41. Miozzi, M., Jacob, B., Olivieri, A. Performances of feature tracking in turbulent boundary layer investigation, Experiments in Fluids, 45 (4), pp. 765-780 (2008).

42. Cowen EA, Monismith SG. A hybrid digital particle tracking velocimetry technique. Experiments in Fluids;22(3):199-211 (1997)

43. Besalduch, L.A., Badas, M.G., Ferrari, S., Querzoli, G., Experimental Studies for the characterization of the mixing processes in negative buoyant jets, EPJ Web of Conferences, 45, art. no. 01012 (2013)

44. Besalduch, L.A., Badas, M.G., Ferrari, S., Querzoli, G., On the near field behavior of inclined negatively buoyant jets, EPJ Web of Conferences, 67, art. no. 02007 (2014)

45. Harris, C. \& Stephens, M. A combined corner and edge detector. In: Proceedings of the 4th Aivey Vision Conference, Manchester, 147-151, 1988

46. La Porta A, Voth GA, Crawford AM, Alexander J, Bodenschatz E, Fluid particle accelerations in fully developed turbulence. Nature 409:1017-1019 (2001)

47. Voth GA, La Porta A, Crawford AM, Alexander J, Bodenschatz E, Measurement of particle 
accelerations in fully developed turbulence. J Fluid Mech 469:121-160 (2002)

48. Mordant N., Pinton J.-F. and Michel O., Timeresolved tracking of a sound scatterer in a complex flow: nonstationary signal analysis and applications, Journal of the Acoustic Society of America, 112(1), 108-118, (2002).

49. Ferrari, S., Rossi, L., Particle tracking velocimetry and accelerometry (PTVA) measurements applied to quasi-two-dimensional multi-scale flows, Experiments in Fluids, 44 (6), pp. 873-886 (2008).

50. Lardeau, S., Ferrari, S., Rossi, L., Threedimensional direct numerical simulation of electromagnetically driven multiscale shallow layer flows: Numerical modeling and physical properties, Physics of Fluids, 20 (12), art. no. 127101, (2008)

51. Ferrari, S., Rossi, L., Vassilicos, J.C., Acceleration measurements in turbulent-like flows, Advances in Turbulence XI - Proceedings of the 11th EUROMECH European Turbulence Conference, pp. 485-487 (2007).

52. Ferrari, S., Kewcharoenwong, P., Rossi, L., Vassilicos, J.C., Multi-scale flow control for efficient mixing: Laboratory generation of unsteady multi-scale flows controlled by multi-scale electromagnetic forces, Solid Mechanics and its Applications, 7, pp. 267-272 (2008).

53. Ran B. and Katz J., Pressure fluctuation and their effect on cavitation inception within water jets, Journal of Fluid Mechanics, 262, 223-263, (1994).

54. Liu X. and Katz J., Instantaneous pressure and material acceleration measurements using a fourexposure PIV system, Experiments in Fluids, 41, 227-240, (2006).

55. Gregory J.W., Sakaue H., Liu T, and Sullivan J.P., Fast Pressure-Sensitive Paint for Flow and Acoustic Diagnostics, Annual Review of Fluid Mechanics, Vol. 46: 303-330 (2014) 